\title{
The Molecular Interactome of the Centriole, Cell Cycle and Ciliary Proteins Modulates Cell Mass Growth and Structural Organization During Development in Metazoans
}

\author{
Roopasree O.J. ${ }^{2}$, Adivitiya ${ }^{2}$, Soura Chakraborty ${ }^{2}$, Suneel Kateriya ${ }^{2}$ and Shobi Veleri ${ }^{3, *}$ \\ 1 Agroprocessing Technology Division, CSIR-National Institute of Interdisciplinary Science and Technology, \\ Thiruvananthapuram, Kerala and Academy of CSIR, New Delhi, India; roopasreeoj@gmail.com \\ 2 Laboratory of Optobiology, School of Biotechnology, Jawaharlal Nehru University, New Delhi, India; \\ skateriya@jnu.ac.in \\ 3 Drug Safety Division, ICMR-National Institute of Nutrition, Hyderabad, India; shobi.veleri@icmr.gov.in
}

\begin{abstract}
Metazoans have an elaborate and functionally segmented body. It evolves from a single cell by systematic divisions. Metazoans attain structural complexity with exquisite precision, which is a molecular mystery. The indispensable role of centrioles in cell division and ciliogenesis can shed insight into this riddle. Cell division helps in growth of the body and is a highly regulated and integrated process. Its errors cause malignancies. The cell mass is organized during organogenesis. Prior to it, the centrioles are retrieved from the cell cycle to initiate ciliogenesis. The cilia-modulated developmental signaling pathways elaborate the body plan. The secluded compartment of the cilium reduces noise during signaling and is essential for a precise body plan development. The dysfunctional centrioles and cilia can distort body plan. Thus, centriole has a dual role in growth and cellular organization. This concept review analyses the comprehensive interactome and the key domain features (like C2 domain) of molecules which connect and disarm the centriole from the cell cycle and ciliogenesis by switching on or off the essential regulators of the pathways. The concentration of these signaling pathways at the centriole reinforces the hypothesis that centriole is the molecular workstation to carve out structural design and complexity in metazoans.
\end{abstract}

Keywords: Metazoa; body plan; developmental pathways; limb development; Sh; centriole; cell division; cilia; centriole gene expression

\section{Introduction}

Metazoans are highly evolved organisms with organized cellular structures that have compartmentalized signaling and functions. Their organs are an amalgamation of the individual cells. The metazoan body layout follows a precise plan embedded in the genetic blueprint $[1,2]$. This blueprint is present in all the body cells, however, all the cells are not the same [3]. The differentiation of cells during development helps in achieving different functions leading to the development of multi-functional tissues within the body [4]. Thus, a magnificent body plan of the metazoans is laid out. To accomplish this goal, cells must regularly perform dual roles. First, the cell mass must be increased by cell division. Next, the cell mass must be molded to build an organized structure of these cells. This requires intra- and intercellular signaling coordination. Furthermore, the two functions of a cell must be offset to avoid any conflict of signaling. How these two events are interspersed, integrated and regulated at the subcellular level is not delineated.

Grown cells are used for the expansion of the structural configuration of an organism. The process of cell division may be regulated and contained within the cell [5]. On the contrary, the structural contour of an organism requires coordination of intracellular signals in tune with 
extracellular signals from the milieu exterieur. The cells have receptors and antenna like cilia for sensing the internal and external signals [6,7]. The receptors are mainly for metabolic activities occurring in both dividing and non-diving cells.

Cilia, unlike metabolic receptors, are uniquely present only in non-dividing cells. They are found in most of the cells during embryogenesis and are also essential for the functioning of the sensory organs and vital organs in the human body. Cilia are essential antennae for sensing external cues during development. They host and coordinate most of the developmental pathways [8]. Therefore, cilia have been utilized as antennae for sensing morphogenetic gradient signal transduction during embryogenesis. This special role for cilia is included during elaboration of structural organization and is excluded in a dividing cell. However, how the centriole mediated signaling for cell division and ciliogenesis mutually excluded is not deciphered. This clearly requires the coordination of intra- and extracellular signaling pathways triggering the initiation and halt of cell division. Similarly, the assembly and disassembly of cilia require signaling mechanisms during development and its dismantling, respectively. The mass growth and repair of wearing out tissue require a constant supply of the cells. This is brought about by the division of existing cells. How the molecular signaling events are linked and coordinated? It is not clear. Here, we conceptualize the critical and dual role of the centriole as a pivot for integrating molecular signaling (Figure 1). It links both cell division and cilia formation, a structure utilized in body plan elaboration. The centriole is essential for cell division to increase cell mass and also plays a key role as a specialized conduit for signaling pathways during organogenesis. These two events are mutually exclusive because the centriole cannot be simultaneously involved in both the cellular events. Thus, the centriole first generates cell mass, then steps out and switches off the event before switching over to develop the cilium. The cilia then coordinate molecular signaling for organizing the generated mass of cells to configure the structural layout of the body.

\section{Centriole serves as a pivot for cell cycle and ciliogenesis}

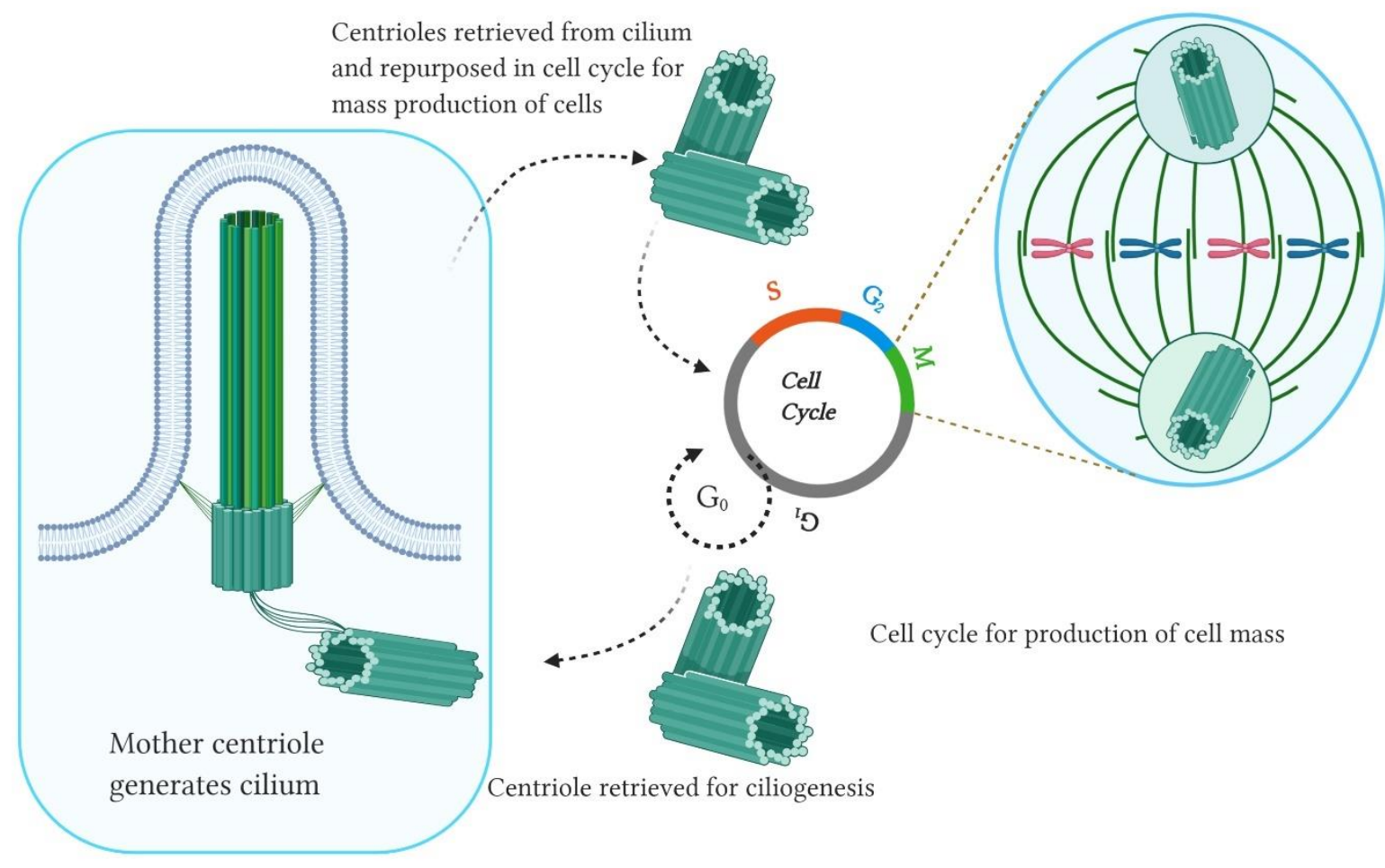

Figure 1. Dual role of centriole in ciliogenesis and cell cycle. Centrioles play important dual roles in cell cycle progression and ciliogenesis in interphase. G1 phase of cell cycle is significant for the cell mass production. Primary ciliogenesis ensues when a cell exits cell cycle and moves into a quiescent 
phase (G0 phase). In this phase, mother centriole templates microtubule assembly and initiates ciliogenesis. Primary cilium acts as a signaling hub in a differentiating cell. In mitotic phase, centrioles organize the spindle poles and centriole generated spindle fibers assist in separation of chromosomes to the opposite poles of dividing cell. [Created with BioRender.com].

In the current article, we review the basics of two events, cell division and ciliogenesis, for which the centriole is the central and essential component. Then, we examine the molecules that regulate cell division and ciliogenesis, with emphasis on the key molecules signaling through the centriole as a hinge for switching on and off of these events. Finally, we critically analyze a set of key proteins associated with the centriole for regulating cell cycle and ciliogenesis by post-translational modifications.

\section{The Cell Cycle and its Regulators}

Cell division is an essential and tightly regulated event with multiple check points in metazoans. The uncontrolled cell division causes cancerous growth instead of a defined development of the layout in the blueprint [9]. The cell cycle is a precisely timed event and comprises of four main phases: G0/G1, S, G2 and M. The G1 and S phases are involved in the preparation for cell division while quality control of the duplicated chromosomal material occurs during the G2 phase. During the M phase, the separation of chromosomes and division of the cytoplasm (cytokinesis) culminates in cell division.

Mitotic division ensures the production of two equal and healthy daughter cells. What triggers the onset of cell division is poorly understood. However, it is obvious that during the early development and growth of an organism the cells receive metabolic signals to initiate division for increasing the cell mass [5]. Cellular signalling is often mediated by switching the structural configuration of proteins by the addition (phosphorylation) or removal (de-phosphorylation) of phosphate groups and other post-translational modifications (PTMs) [10] [11]. This is the basic mechanism of metabolic regulation [12]. The same mode is employed to switch on and off the steps of the cell cycle. Several centriole core proteins and centriole associated proteins are involved in the cell cycle and are subject to phosphorylation and other PTMs so as to regulate various stages of the cell division (Figure 2).

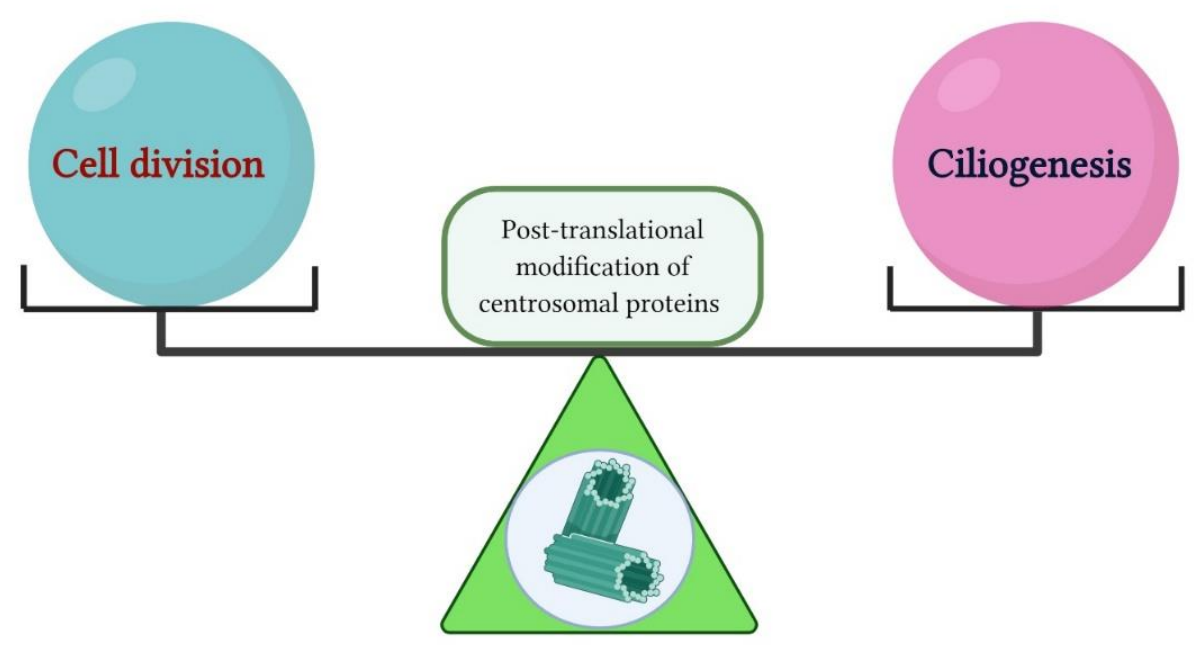

Figure 2. Post translational modifications of the centriolar proteins balance the production of cell and its differentiation. Signalling through the centriole channels the cell fate for production of cell mass or differentiation of the cells mediated by cilia. [Created with BioRender.com]. 
The role of protein phosphorylation in the cell cycle is demonstrated by a peak in the phosphorylation profile during mitosis which overlaps with the chromosomal and cytoskeletal reorganization [13, 14]. Thus, mitotic protein kinases are critical proteins for mitotic progression. The expression and function of these kinases at the precise location and its timing are essential for an error free chromosome segregation and maintenance.

The family of conserved serine/threonine kinases such as Cyclin Dependent Kinases (CDK), Aurora kinase and Polo Like Kinase (PLK), are the main classes regulating the cell cycle [15]. In $\mathrm{CDK}$, cyclin functions as a regulatory subunit which imparts substrate specificity while the cyclin dependent kinase (CDK) operates as the catalytic subunit [16]. The genome of mammals encodes 29 cyclins and 20 CDKs [16]. Mammalian CDKs are evolutionarily divided into two groups, namely cell cycle regulators and transcriptional activators, that are further subdivided into eight families. CDK1, -4 and -5 subfamilies are primarily involved in cell cycle progression [17]. CDK1 is deemed the master in cell cycle regulation and is indispensable for initiating mitosis in the eukaryotes. The levels of CDKs oscillate during the cell cycle in tune with its phase progression. Different combinations of Cyclin-CDK complexes can initiate different phases of the cell cycle. Cyclin D is a G1 phase cyclin which activates the corresponding CDKs (CDK4 and CDK6) in response to growth factor signals. Activation of Cyclin D-CDK4 and -CDK6 complexes promotes the expression of the genes required for DNA replication. The initiation of centriole duplication and its continuation into the $S$ phase necessitates Cyclin-E-CDK2-kinase and-Cyclin-A-CDK2 kinase activity, respectively [18-20]. Cyclin $\mathrm{E}$ possesses a centrosomal targeting domain that is required for entry into the $\mathrm{S}$ phase independent of CDK2 [21]. CCP110 and Nucleophosmin (NPM/B23) are the substrates of Cyclin-E-CDK2 kinase. Human CP110 is expressed during the G1-S transition and localizes to the centrosomes. It has critical function in duplication of the centrosome. Anomaly in phosphorylation of CP110 leads to polyploidy and genomic instability [22]. Nucleophosmin (NPM/B23) binds specifically to unduplicated centrosomes and upon its phosphorylation disengages from the centrosome, initiating the duplication of centrosome [23]. We have constructed the molecular interactome of proteins regulating the cell division and ciliogenesis (Figure 3). It contains the three key proteins mentioned, CDK2, PLK4 and CP110.

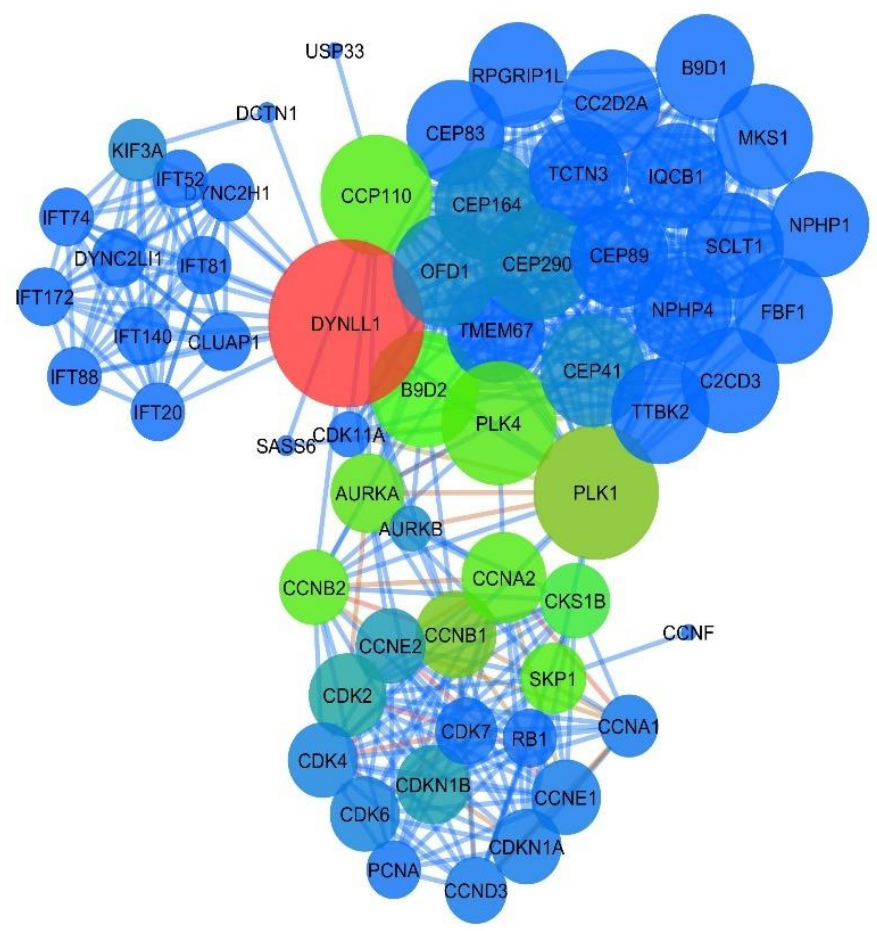

Figure 3. The protein interactome concentrated on centriole involved in cell division and ciliogenesis, including its sub-functional unit intraflagellar trafficking protein. The interactome of the proteins has three clusters involved in ciliogenesis, cell cycle and centrosomal maintenance. Five genes coding protein with high degree, act as hub of the network and one gene with highest betweenness 
centrality value act as a bottleneck gene. Hubs: DYNLL1 ( $k=40)$, PLK1 $(k=31)$, PLK4 $(k=28)$, CP110, also called CCP110; (k=27), B9D2 (k=26) Bottleneck gene: DYNLL1 (B.C.= 0.37271065). Number of nodes: 59, number of edges: 503, average node degree: 17.1 , avg. local clustering coefficient: 0.883 , expected number of edges:47, PPI enrichment p-value:<1.0e-16, Highest confidence (0.900).

CDKs mainly regulate the activity of RNA polymerase II by phosphorylating its C-terminal domain, which is an essential step during transcription [17]. In addition to a role with cyclin, CDKs are also controlled by their phosphorylation status to activate or inhibit their function. Phosphorylation of threonine near the active site of the CDK enzyme results in its activation which is mediated by the CDK activating kinase (CAK). In contrast, the phosphorylation of tyrosine at position 15 and threonine at 14 in CDKs by Wee1 kinase and Myelin transcription factor 1(MYT1) inhibits the function of CDKs. Thus, changes in the phosphorylation of the cyclins at different sites are essential for differential regulation of the cell cycle at different phases [17].

Another level of regulation is achieved by dephosphorylation of the kinases. It is mediated by a highly conserved phosphatase, CDC25, that activates the CDKs [24]. PLK1 phosphorylates CDC25 for its activation and translocation into the nucleus [25]. CDK activity is counter balanced by dephosphorylation by Protein Phosphatase 2A (PP2A) [26]. These multiple level checks and balances are employed to ensure that CDKs are only active in defined spatio-temporal manner.

Similarly, cyclins are also under post-translational control to ensure the proper sequence of events during the cell cycle. Cyclins are only expressed in the specific phase of the cell cycle that they trigger and are absent in the other phases. Various transcriptional factors regulate the expression of these cyclins. The G1 cyclins are present throughout G1 and are expressed at increased levels in response to growth factors. Near completion of G1 phase, Cyclin E joins CDK2 and the complex is activated later [for review see [27]] and that completes the phosphorylation of Rb (pocket proteins). It triggers expression of the genes that perform DNA replication/repair and mitosis. This eases passage through the bottle neck at the boundary of the G1/S phases. Later, CDK2 takes over as an important participant in $S$ phase.

A-type cyclins are mainly regulated by two families of CDK inhibitors during normal and stress conditions like DNA damage and telomere dysfunction. First is INK4 family that blocks D-type cyclin activity by specifically binding to CDK4 and CDK6. Second, the Cip/Kip (CDK Interacting Protein/Kinase Inhibitory Protein) family inhibits CyclinE-CDK2, Cyclin A-CDK2, Cyclin A-CDK1, as well as Cyclin B-CDK1 activity [28, 29]. Cyclin A-CDK1 activity is necessary for the beginning of prophase from G2 to M boundary [30]. Ultimately, functioning of Cyclin B-CDK1 complex completes mitosis [31].

The Anaphase Promoting Complex (APC) induces ubiquitination of cell cycle factors for degradation. The APC mechanism is a critical means for cells to precisely regulate progression through various phases of the cell division. Thus, the APC coordinates phase setting of mitosis at anaphase entry, anaphase progression, exit of mitosis and G1 phase [32]. The level of cyclins is negatively regulated by the ubiquitin ligases [Skp-Cullin-F-Box (SCF) and APC/C complex] for polyubiquitination and subsequent degradation of cyclins in the proteasome.

Cyclin E accumulates during the G1-S transition phase and its deregulation induces tumorigenesis $[33,34]$. Regulation of Cyclin E levels occurs via the Ubiquitin Proteasome System (UPS) that mediates its proteolysis by the F box protein (human CDC4), which provides specificity to the SCF-FBX7 E3 ubiquitin ligase [35, 36]. Cyclin E can either phosphorylated by CDK2 activity or is phosphorylated by Glycogen Synthase Kinase 3b [36, 37]. CDC4 exhibits a high binding affinity to a phosphopeptide motif, CDC4 Phosphodegron (CPD ) within its substrate such as Cyclin E [38]. The PLK2 phosphorylates $\mathrm{F}$ box protein (Fbxw7) at few conserved serine residues and weaken the latter. This causes an increase in level of Cyclin E thereby inducing centriole duplication [39].

Cyclin F is first member of the F box protein family [40]. It does not bind and activate any CDK but peaks during the G2 phase [41]. It binds to CP110 at the centrioles. As a consequence CP110 is targeted for degradation by the proteasome. This process ensures mitotic fidelity and genome integrity [42]. A de-ubiquitylating enzyme (DUB), USP33, can also regulate CP110 levels thereby regulating centrosome biogenesis. These studies exhibit that an equilibrium between ubiquitylation 
and de-ubiquitylation regulates the levels of a key protein involved in maintaining centrosome homeostasis [43]. The DUB, USP9X, stabilizes the Peri Centriolar Material 1 (PCM1) protein and maintains centriolar satellite integrity [44]. Mutation and loss of SUMO encoding gene causes chromosomal aberration. Recently, global proteomic profiling of SUMO, during the cell cycle, deciphered that SUMO modulated function of the cell cycle protein and associated transcription factor (TF) Fox M1. It undergoes SUMOylation during G2 and M phases [45]. SUMOylation of ARL 13, a cilia microtubule associated small GTPase, is involved in ciliary trafficking of the membrane proteins [46]. However, whether SUMOylation plays any role in IFT machinery is unknown. Is there any link between ubiquitination and sumoylation of the cilia machinery? These are unexplored questions to be addressed.

The proteasome complex (SCF) degrades G1/S transition phase cyclins and APC/C complex degrades $\mathrm{S}$ phase and mitotic cyclins, It promotes exit from mitosis. CDK inhibitors (CKIs) are other set of molecules that inhibit Cyclin-CDK. They play an important role in G1/S transition phase [47]. As stated earlier, CDKs also have a regulatory function outside the cell cycle. For example, CDKs play an additional role in the control of centrosome duplication [17].

The PLKs play varied but crucial roles during mitosis, cytokinesis and centriole duplication [48]. Mammalian cells express five PLKs (1-5) [49]. PLK 1-4 have kinase domains, which are utilized for fine tuning cell cycle checkpoints, mitosis, cytokinesis, and for initiating duplication of centriole $[48,49]$. They have descended from a PLK1-like ancestor in fungi, ciliates, and are also seen in the higher animals [49]. PLK1 is responsible for centrosome maturation, formation of normal mitotic spindles and cytokinesis [50-53] and it has a high degree of function on centriole (Figure 3, PLK1). PLK2 has been shown to be involved in cell cycle progression from G1 to $S$ phases and in embryonic development of mice [54]. In addition, its role has also been implicated in centriole duplication in the mammalian cells $[55,56]$. PLK3 is recruited to combat DNA damage and spindle disassembly process $[57,58]$. PLK4 (Sak) is a main modulator of centriole duplication and has high degree of functional role in centriole (Figure 3; PLK4). It is localized in the centriole at the peripheral side towards the proximal ends [59] and co-localizes with several proteins involved in centriole biogenesis. This indicates that PLK4's role is in centriole biogenesis, which initiates at the proximal end of the centriole. PLK4 tightly controls the fixed number of centrioles in a cell that is essential for chromosomal integrity and genetic stability. Abnormal levels of PLK4 results in multiple centrioles and genomic instability, which causes tumorigenesis [60-63]. Plk4 has been shown to be highly expressed in colo-rectal and hepatocellular carcinoma tissues of the patients [64, 65]. Additionally, it has been shown to accelerate the incidence of cancers in liver and lung of Plk4+/- knock out mice and drive genetic instability resulting in tumorigenesis in Drosophila [65] [66].

Thus, regulation of PLK4 expression is critical for the maintenance of genomic stability. PLK4 has a short half-life and undergoes ubiquitination that promotes its degradation by $26 \mathrm{~S}$ proteasomal machinery [67-70]. The SCF/Slimb ubiquitin ligase carries out PLK4 degradation and its absence in Drosophila resulted in amplification of centrosome [68, 70]. Inactivation of $\beta$-Trcp1 (F box protein) in mice was shown to result in centrosome over duplication, multipolar metaphase spindles, and misaligned chromosomes [71]. PLK4 auto-phosphorylates that results in autoregulation of its stability to prevent centrosome amplification [72-74]

Aurora kinases that work on centrioles to switch its function between cell cycle and ciliogenesis are expressed in proliferating cells and are overexpressed in a few tumor cells [75]. Aurora kinases regulation, like PLKs, follow a similar mechanism of phosphorylation and ubiquitinylated proteasome degradation. Temporally, expression of Aurora A precedes Aurora B. Spatially, Aurora A seen associated with centrosomes and the spindle apparatus in prophase till telophase. This suggested a role for Aurora A in maturation of centrosome and integrity of mitotic spindle. TPX2 (Targeting protein for $\mathrm{Xklp2}$ ), an important component in spindle apparatus is required for targeting Aurora A kinase to the spindle [76]. Aurora B is expressed during anaphase and telophase at the mid-zone and the post-mitotic bridges, respectively. It is essential for kinetochore-microtubule attachment and avoid destabilizing errors. Inner centromere protein (INCENP) is a partner of Aurora B for cytokinesis [77]. These INCENP forms the Chromosomal Passenger Complex (CPC) 
with Boreali and Survivin. In eukaryotes, CPC proteins are implicated in chromosome condensation, congression, segregation, the spindle dynamics and cytokinesis [77].

Aurora kinases act upon PLK and interact with the centrioles. The critical role of PLKs for mitotic entry is known [78]. During mitotic entry, PLK is activated with phosphorylation by Aurora A in C.elegans [79] and Aurora B in Drosophila [80]. The phosphorylation site is an evolutionarily conserved Thr210 in the activation loop of human PLK1 [81, 82]. Phosphorylation of PLK induces its trans-localization from the cytosol to the nucleus, It might control genes in the nucleus to promote mitotic entry. Taken together a plethora of molecules tightly control the cell cycle progression as pre-programmed in genetic blueprint. Most of these regulators are pivoting on the centriole as the locus of action (Figure 3).

\section{Centriole's Dual Role: A Pivot for Expanding Cell Mass and Organizing Metazoan Structural Layout}

Centrioles are central to cell division. They accurately segregate the duplicated chromosomes for chromosomal integrity and thereby ensure propogation of the blueprint for cellular function and structural organization. During prometaphase, the chromosomes come to lie at the mid point between the two spindle poles, a process called congression, which is coordinated by the several microtubule-based regulators used for microtubule assembly and disassembly. The microtubule extension is organized from a locus, the Micro-Tubule Organizing Center (MTOC), which has the centrioles in the middle with gamma tubulins [83]. The centrosome is the main MTOC in animal cells. During mitosis, cells organize their two centrosomes at each pole to form bipolar spindles that extend microtubules to accurately segregate one copy of the duplicated chromosomes to each daughter cell. Thus, centrioles ensure spindle formation and proper segregation of the chromosomes for fecundity of the cell division. The expanded line of cells is utilized to configure the structural layout of the organism. This is mediated by developmental signaling pathways. It requires discrete signaling hubs in the cells for efficacy and efficiency [84, 85].

The centrioles have themselves evolved to accommodate a dual role for this. The expanded cell mass must be utilized first for a milestone in development, before producing more cells for the next milestone. The same microtubular ultrastructure of centrioles used for chromatid separation is re-deployed to create a discrete signaling hub for multiplex developmental signaling pathways, in order to organize the expanded cell mass. The centriole is retrieved from the cell cycle and utilized for microtubule extension during the ciliogenesis. However, how these two events are alternatively triggered is unclear. To gain a mechanistic insight on this one can examine the signaling during centriole biogenesis and ciliogenesis [86].

The inherited centriole in a cell is called the mother centriole. It duplicates to form a daughter centriole in a newly formed cell to maintain the configuration of two centrioles per cell. The basic structure of a centriole is composed of a 9-fold symmetry of triplets made of gamma-tubulins. The mother centriole acquires additional structures to configure the basal body during ciliogenesis. It accumulates a myriad of proteins, the pericentriolar material (PCM), which is the core of microtubule nucleation $[87,88]$, from where microtubule astral organization sprouts.

\subsection{Centriole Duplication is an Event Inside Cell Cycle for Mass Production of Cells}

The biogenesis of the centriole occurs once per cell cycle. However, the real triggering signal for centriole duplication remains unidentfied Upon receiving a putative signal by the parental centriole at its proximal end a procentriole is assembled towards the G1-S transition phase. The procentriole elongates during transition of $S$ phase to the G2 phase. By M phase, the procentriole acquires a size approximating that of the mother centriole. The older centriole develops appendages in late G2 phase/early $M$ phase whereas the daughter centriole loses its appendages during this time [89]. Until late mitosis, the newly formed centriole is tightly associated with the mother centriole. Each procentriole separates from its parental centriole in the late $M$ phase, a process termed as disengagement. How this process is regulated is not well understood. Investigating the molecular changes during biogenesis and disengagement of centrioles have shed some insights on the process. 
PLK4 plays a key role in controlling centriole biogenesis (Figure 3). It is transcriptionally activated, and its level is maximum during mitosis [74]. The accumulation of PLK4 at the proximal end of the parental centriole marks the beginning of centriole duplication [59, 74]. The absence of PLK4 results in mitotic arrest at the grastrulation stage of mouse embryogenesis and is a cause of embryonic lethality. The recruitment of PLK4 to centriole requires action of cell cycle regulator [CDK11p58 [90]] and centriolar proteins CEP192, CEP152 [91]. This suggests that a pathway mediated through CDK11p58 is activated before duplication of the centriole that involves PLK4.

The duplicated centriole's disengagement requires the involvement of cysteine proteases, Separase and PLK1, and that is the 'license' for centriole duplication [92], which is also essential for ciliogenesis. It supply all these ingredients in the correct amount because excess of PLK4 is associated with multiple centrioles, a condition seen in tumor cells [72]. PLK4 turnover is regulated via ubiquitination and degradedation by the $26 \mathrm{~S}$ proteasome [67]. If this is inhibited P-PLK4 gets accumulated [74]. Thus, PLK4 is a key molecule determines the fixed number of centrioles in the cellsand it has far reaching implication for genomics stabiilty besides producing cell mass [90].

\subsection{Mother Centriole Mediated Ciliogenesis: the First Step of Building a Signaling Hub Required for Elaborating the Structural Layout of the Organism}

The centrioles are no longer required for chromosomes in the G0 cell. Hence, the centrioles are retrieved and repurposed for developing a signaling hub to utilize the newly formed cells for developing the layout of the body. This function largely occurrs in the signaling hub hosted by the cilia, which are extensions of microtubules originating from the mother centriole. A cilium is a secluded compartment of the plasma membrane extension. Inside this compartment most of the fundamental developmental pathways are hosted [8].

The transition of the centriole to the mother centriole occurs in a few steps. The nine fold symmetry of the centriole is nucleated by recruitment of the SAS6 protein, which is a part of the protein interactome (Figure 3), and important for triple 9 MTs structure of the basal body [93, 94]. First, the centriole is translocated to the cell periphery as opposed to a central position for the centriole pairs in a dividing cell. Then, the mother centriole develops additional structures like distal appendages to anchor to the peripheral plasma membrane. To dock cargos for building and maintaining cilia the mother centriole has subdistal appendages. It docks microtubule tracts carrying the cargos. These appendages are absent on daughter centrioles [95, 96]. The pair of centrioles (mother and daughter) remain tethered by interconnecting fibers during ciliogenesis. Many proteins are involved in the distal and subdistal appendages. Analyzing how these structures are developed and what are the signaling triggers will give insight on the process transforming the centrioles from cell cycle to ciliogenesis.

Cilia are dynamic structures and their disassembly is a sign of re-entry into the cell cycle. Various proteins are expressed coordinately and in a hierarchical manner to control the assembly and disassembly of cilia. The addition of appendages to the mother centriole transforms it to the basal body that paves the foundation for assembling the cilia. Cilia assembly is a well regulated process which requires the phased timely organization of negative and positive players at the basal body. Accumulation of proteins around the mother centriole nucleates ciliogenesis (Figure 3).. PLK4 is a key component in centriolar satellite organization During the G1 phase, it carries out the phosphorylation of PCM1 at the conserved S372 residue thereby promoting its dimerization, which is essential for the interaction of PCM1 with other centriolar satellite components such as hMsd1/SSX2IP, BBS4, and CEP290 thereby enhancing the localization and aggregation of the centriolar satellites [97]. The integrity of centriolar satellite organization is critical for error free ciliogenesis. PLK4 depletion could disperse centriolar satellites that ultimately end in loss of cilia itself. This uproots many developmental pathways like Wnt, PDGFR $\alpha$, Sonic Hedgehog (Shh). The Shh pathway mis-signalling could even lead to carcinogenesis and other diseases.

The mother centriole characteristically has the distal and subdistal appendages. They are critical structures requiring some of the key proteins for their development [98]. For example, CEP164 maintains the structural integrity of distal appendages, whereas ODF2, CC2DA and Ninein are 
required for integrity of subdistal appendages [99]. Further, several kinases exert a major role in cilia assembly and maintenance. Serine threonine kinases like TTBK2 and MARK4 positively regulate cilia assembly. TTBK2 is concentrated at the basal body [100], it removes CP110 and accumulates IFT complexes for maintenance of cilia. Pitchfork (PIFO) works upstream of Aurora A Kinase and is present in the embryonic node, neural tube floor plate, the apical ectodermal ridge and growth zone of the embryonic limb bud [101]. PIFO along with Trichoplein activate Aurora A kinase, which in turn activates Histone Deacetylase 6 (HDAC6) [102]. PLK1 interacts with canonical Wnt signaling protein Disheveled and together activates HDAC6. The depletion of trichoplein leads to ciliation. CEP97, a centrosomal protein, and a member of the microtubule depolymerising kinesin family of protein, KIF24, recruits CP110 to the mother centriole and positively regulates ciliogenesis.

The cilia disassemble prior to cell cycle re-entry. The basal body must be released from the membrane and migrate near the nucleus to form the spindle polar body, a critical apparatus for cell division [103, 104]. Cilia disassembly before cell cycle re-entry can occur via two different mechanisms, deciliation or resorption. Deciliation occurs in response to environmental, and chemical (ROS) stress and it is observed in cultured mammalian cells [105]. PIFO, Aurora A and HDAC6 in sequence work to achieve the deacetylation of the microtubules of the axoneme. This process facilitates the resorption of cilia [106]. Aurora A solely can trigger quick ciliary resorption and destabilization of the ciliary axoneme. KIF2A is a protein which controls spindle assembly and possesses microtubule depolymerizing activity. It is seen at the basal body and it might depolymerize centrosomal microtubules, which could disrupt trafficking of cargos into the cilium [107]. TCTEX-1, a subunit of dynein motor, that is involved in retrograde trafficking in cilium and is accumulated in basal body before the $S$ phase that apparently facilitates cilia disassembly [108]. Ciliary retraction also requires the Human Enhancer of Filamentation1 (HEF1) along with Aurora A andHDAC6 and they are in cascading reaction [109]. The ubiquitin proteasome machinery also regulates cilia formation and maintenance [110]. The non-covalent attachment of the small protein modifier ubiquitin $(\mathrm{Ub})$ to the lysine side chains is catalyzed by the E3 $\mathrm{Ub}$ ligases [111] and the topology of the poly-Ub chains can drive $26 \mathrm{~S}$ proteasome mediated degradation [112]. Disruption in UPS activity at the primary cilium is known to cause ciliopathies. Mutations in the RPGRIP1L cause Joubert Syndrome; RPGRIP1L has a es proteasomal partner PSMD2 and both are localized in the primary cilium [113]. A functional genomic screen using RNAi identified several modulators (36 positive and 13 negative) of ciliogenesis control [114]. Several molecules in the cascade of cAMP signaling, like G-protein coupled receptors (GPCRs), are critical for cilia formation $[115,116]$. The cAMP dependent protein kinase A (PKA) holoenzyme complexes are targeted to the basal body for ciliogenesis. The serine/threonine protein kinase, NEK10, is required for mammalian cilia formation and an increase in cAMP levels causes cilia resorption. This is due to the phosphorylation of NEK10 by PKA that allows ubiquitination by E3 ligase CHIP and degradation. This represents a kinase signalosome at the pericentriolar site that links the cAMP signaling with proteasome machinery thereby fine tuning cilia biogenesis [117]. Thus, many proteins, directly or indirectly associated with the centriole, are sequentially activated or deactivated for assembly and disassembly of the cilium (Figure 3). But still the critical players signalling exit and re-entry of cell cycle are not identified. It may be triggered by the TFs involved in the developmental program overriding the regular cell cycle pattern.

\section{Cilium: An Extension of the Centriole and the Hub for Developmental Signaling Pathways}

In early development, cilia serve as a hub for almost all developmental pathways. It seems logical to host associted cascades within the cilia because they are the extensions of centrioles, which also are involved in expanding the cell mass by cell division. Once enough cell mass has been generated, the next sensible step is to build a structure as per the blueprint of the layout, and is done by deploying the developmental pathways. The cilium can sense the extra cellular signals and coordinate with the intra cellular events. Therefore it gained the nickname antenna of cell [118]. A network of molecules organized in the cilia executes a diverse array of signalling pathways like Shh, Wnt, PCP, BMP, Notch, Hippo, PDGF GPCR, (and other RTKs including FGF), mTOR, and TGF-beta 
[119]. The disruption of cilia mediated signalling like Shh signalling results in severe developmental errors as in Gruber Syndrome, a ciliopathy [120, 121] (Figure 4).

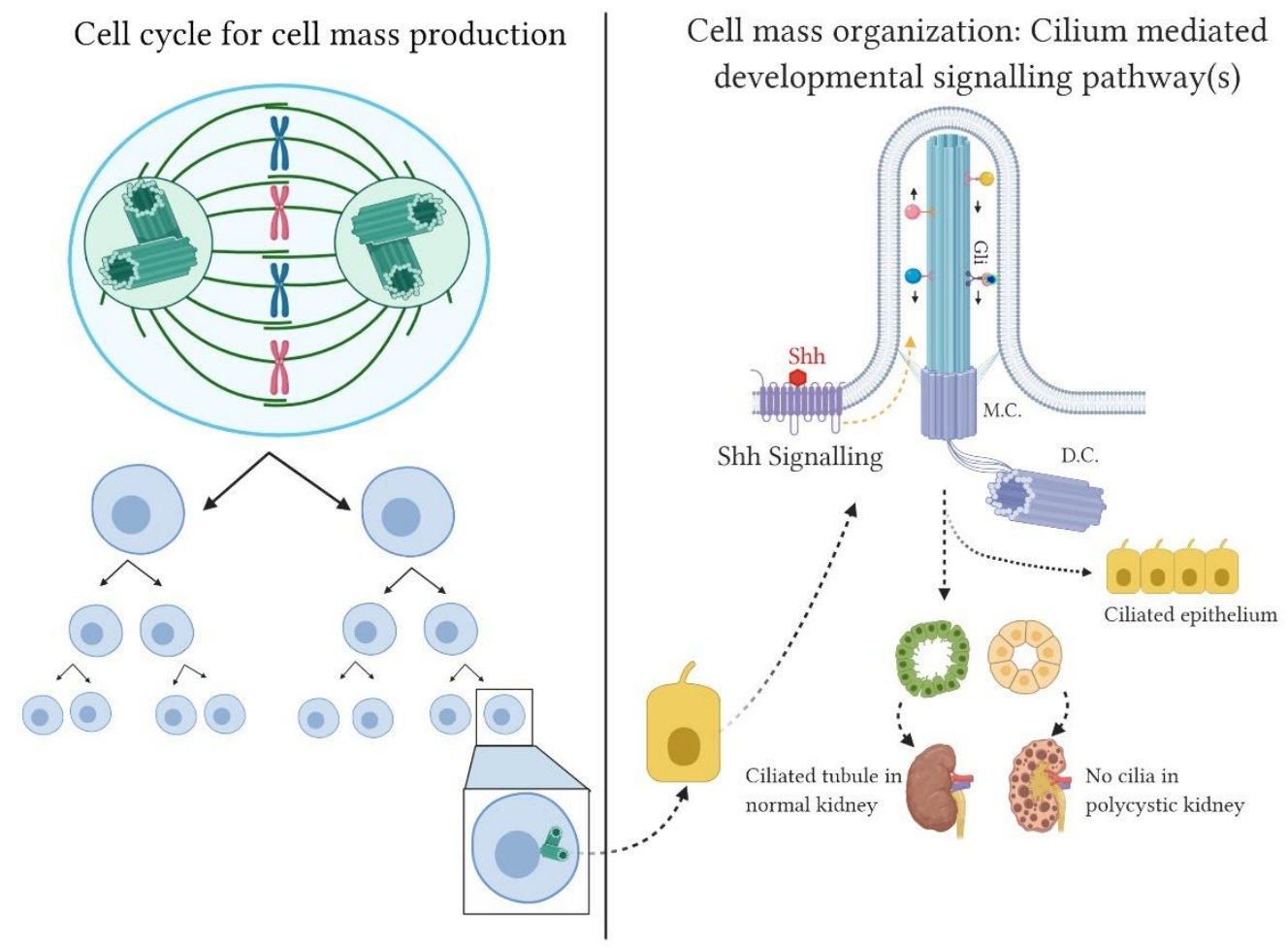

Figure 4. Centrioles role in production of cell mass and cilia and dysfunction of primary cilia results in ciliopathy. Multiple cycles of cell division result in a cell lineage. Centrioles organize the poles of mitotic spindle and assist in the separation of chromosomes during cell division. In growth phase of a differentiating cell, mother centriole (MC) organizes primary cilium, which act as an important signaling hub for different types of developmental signaling pathways. Increased Sonic hedgehog (Shh) signaling is responsible for the cilia function. Cilia growth and cilia maintenance are coordinated by intraflagellar transport (IFT) machinery. Structural or functional dysfunction of primary cilia in kidneys develops polycystic kidney. [Created with BioRender.com].

The role of the cilium in elaborating a structural body plan could easily be understood by the obvious example of digit pattern development of the hand. Cilia mediated developmental pathway aberration results in polydactyly, a salient feature of many ciliopathies like Bardet-Biedl syndrome [122]. Thus, centriole as part of the cilium is the key organelle for coordinating elaboration of the body plan.

There must be a signaling molecule that intersects pathways involved in cell cycle and ciliogenesis, which mediate cell production and organization, respectively. We have examined the theoretical possibilities of such molecule(s) involved in coordinating the cell cycle and ciliogenesis through an interactome analysis (Figure3). Abnormal cell division leads to abnormal growth of tissues beyond the body plan, whereas, the disruption of cilia signaling disrupts organogenesis and results in multi-organ defects that are often seen as congenital developmental defect in ciliopathies.

\section{How Expanded Morphogenic Gradient Signaling in Cell Mass is Routed via Centriole for Building Body Plan}

During metazoan development the cell mass is expanded and organized to generate the body plan engrailed in the genetic blueprint. This is achieved through execution of the developmental pathways (Figure 4). There are many examples to display the role of cilia in developmental pathways that determine body plan. For example, the internal visceral asymmetry achieved by nodal cilia in early embryo and external bilateral symmetry of limbs. However, the role of cilia in body plan development could be easily understood with an obvious example of the digit patterning 
on human hands and feet [123]. The stereotypically ordered position of fingers and number of bones in digits and their patterning happens with amazing precision (Figure 5).

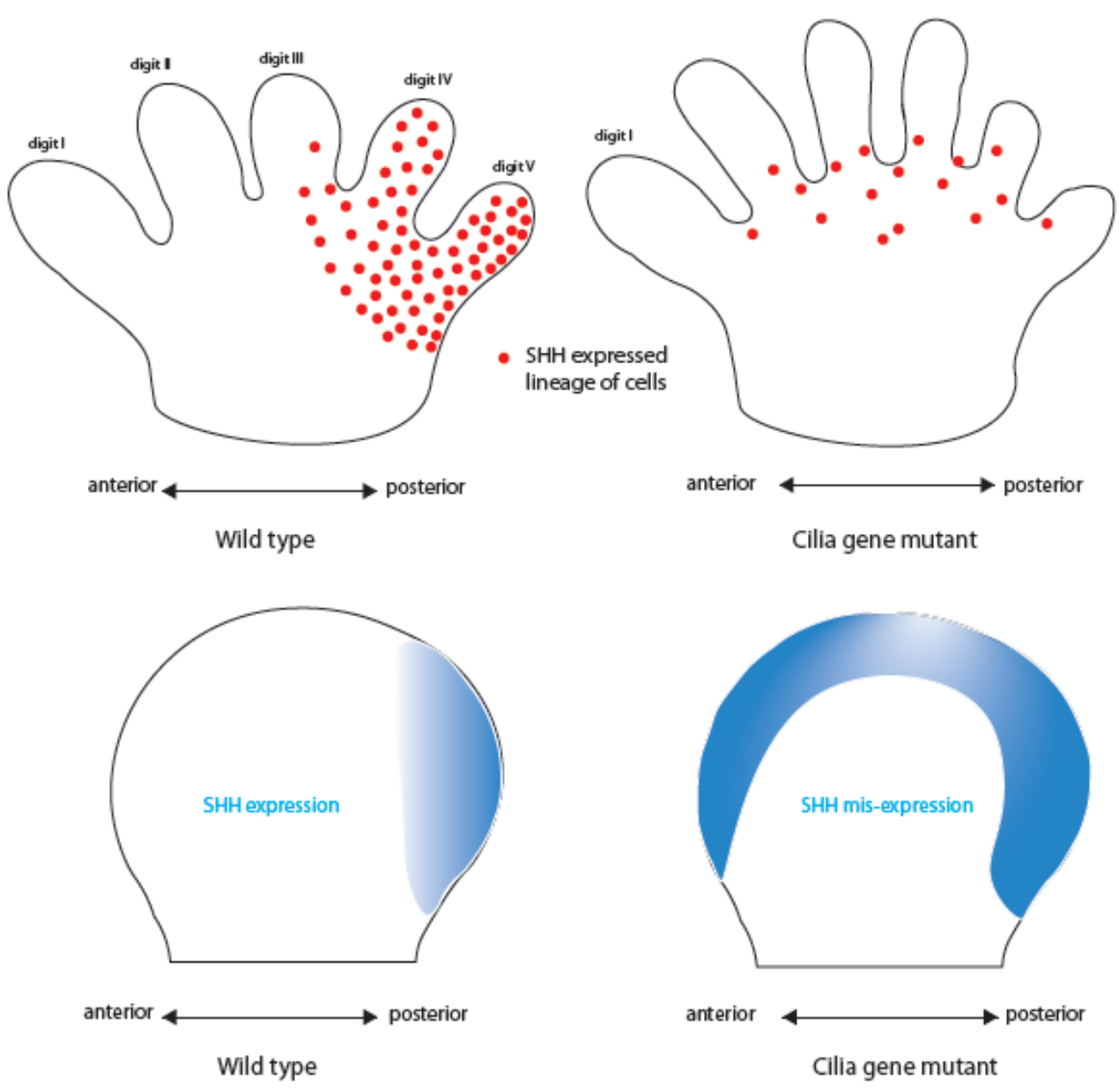

Figure 5. Digit patterning across the anterior-posterior axis of the limb bud. In mammals digit patterning is determined by the gradient of Sonic hedgehog (Shh) signals at the limb bud in early development like at embryonic day 8 (E8). This signaling is mediated through cilia present in the limb bud. Therefore, the lack of cilia results in abnormal signaling of Shh and resultant polydactyly. In normal cilia (left) the Shh signal is maximum at the V digit and minimum signal at the I digit, which becomes the thump. Lack of cilia in the mutants perturbs the gradient signaling (right) and development of abnormal number of digits.

Mammalian primary cilia mediate Shh signaling required for developmental processes like digit patterning. Sonic Hedgehog is a morphogenetic signal secreted by Zone of Polarizing Activity (ZPA) and is essential for the development of a correct set of precisely shaped digits along the anterior-posterior axis of the vertebrate limb bud [124]. The gradient of Shh elicits digit position and its numbers and is also a time-dependent factor. The anterior cells of limb bud arise independent of trigger from Shh and form the digit I (Thumb). The precursors of digit II and anterior half of digit III are specified by early gradient of Shh from the posterior because the digit primordium has no Shh. An interplay of concentration and timed exposure of Shh gradient specifies digit III. The cells with the high level autocrine Shh signals become the digits IVand V. An appropriate long-range Shh signaling is required to specify the whole set of digit identities [123]. Mutations in several ciliary components affects the spread of morphogen gradient shh hence disrupting the patterning of limb bud. When the shh diffusion is higher than normal, the resulting phenotype shows extra digits. Abnormal Shh signaling during limb morphogenesis is a cause for lack of ulna and digits II and V, in vertebrates including humans. This does not affect digit I. 
In mammals, Shh pathway plays a key role in digit patterning based on a highly conserved pentadactyly pattern in limb bud. The digit patterning in the limbs of tetrapods is executed via the precise dynamic action of the secreted factor Shh, it is a highly conserved pathway from flies to mammals and utilized in various places in development, like in neural tube closure [99]. Mutational analysis of genes in this pathway revealed two key factors responsible for digit patterning. First, genes that are a part of developmental pathway like Shh and second, genes associated with the centriole required for ciliogenesis and their defects cause congenital defects like polydactyly and ciliopathy. The position with strongest Shh signal (Figure 5) becomes the digit $\mathrm{V}$ and the position with no signal by default become the thumb/first toe. In limb bud, Shh pathway is routed via the cilia. Therefore, disruption of the Shh pathway due to defective cilia results in abnormal signaling and polydactyly. Mutations in cilia genes could disrupt processing of GLI3 and mis regulation of down streaming TFs thereby leading to polydactyly [125]. Thus, the role of the centriole in structural organization of cells mediated by signaling in cilia is clear. This demonstrates an alternate role for centriole in development other than in production of mass by cell division. Hence centriole is an integrating link for the developmental pathways, through cilia and for cell division. These two events are inter-changed for the development of structural organization in metazoans. The direct role of cilia in structural development during organogenesis is visible by the phenotypes caused by mutations of the centriole associated genes that are required for ciliogenesis. A fundamental cilia gene is required for configuring the mother centriole and is essential for embryogenesis itself [99]. Absence of such gene products resulted in multi-system developmental abnormalities like situs inversus, polydactyly, poly cystic kidney (Figure 4), anormal tissue layering in the retina etc. Null mutation of such fundamental cilia gene hence results in lethality. This demonstrates that the centriole is a pivot for both cell division and body plan development. Each of these steps requires specific signaling pathways and molecular interaction. Therefore, molecular mechanisms must exist associated with the centriole to switch it between cell division and structural development. Understanding the key molecules signaling the switching between cell division and organogenesis via centriole and cilia mediated signaling will provide insight on how cancer is developed. For normal body patterning, the centriole scans the external signal as an antenna and channelizes the molecular morphogenetic gradient signaling pattern for tissue organization. The signaling pathways inside cilia also coordinate each other for proper patterning of the tissues. Thus, centriole demonstrates an alternate role in development other than production of cell mass.

\section{Molecular Regulation and Protein-Protein Interactions are Required for Swapping the Cellular Function of Centrioles in Cell Division and Ciliogenesis}

\subsection{What Could Be the Nature of a Putative Protein Linking Cell Cycle and Ciliogenesis?}

During development, many binary decisions are made by transcriptional factors for committing cell lineage for a particular organ. The TF expression in a subset will be committed for differentiation of a specialized set of cells. If a TF is not expressed in the precursor cells, they follow the default cell lineage fate. Regulated expression of Otx TF commits cells for neural tissue development [126, 127]. Similarly, controlled expression of NRL deviates photoreceptor development configuration from cones to rods [128]. A molecule with domains to sense signatures of a TF is a good candidate for switching the functional role of centriole. Ciliogenesis is mostly seen in the post-mitotic and differentiated cells. Hence, presence of the proteins interacting or controlling TF(s) that determines cell differentiation is a logical entity to be considered for initiating the cellular signaling towards ciliogenesis (Figure 6). 

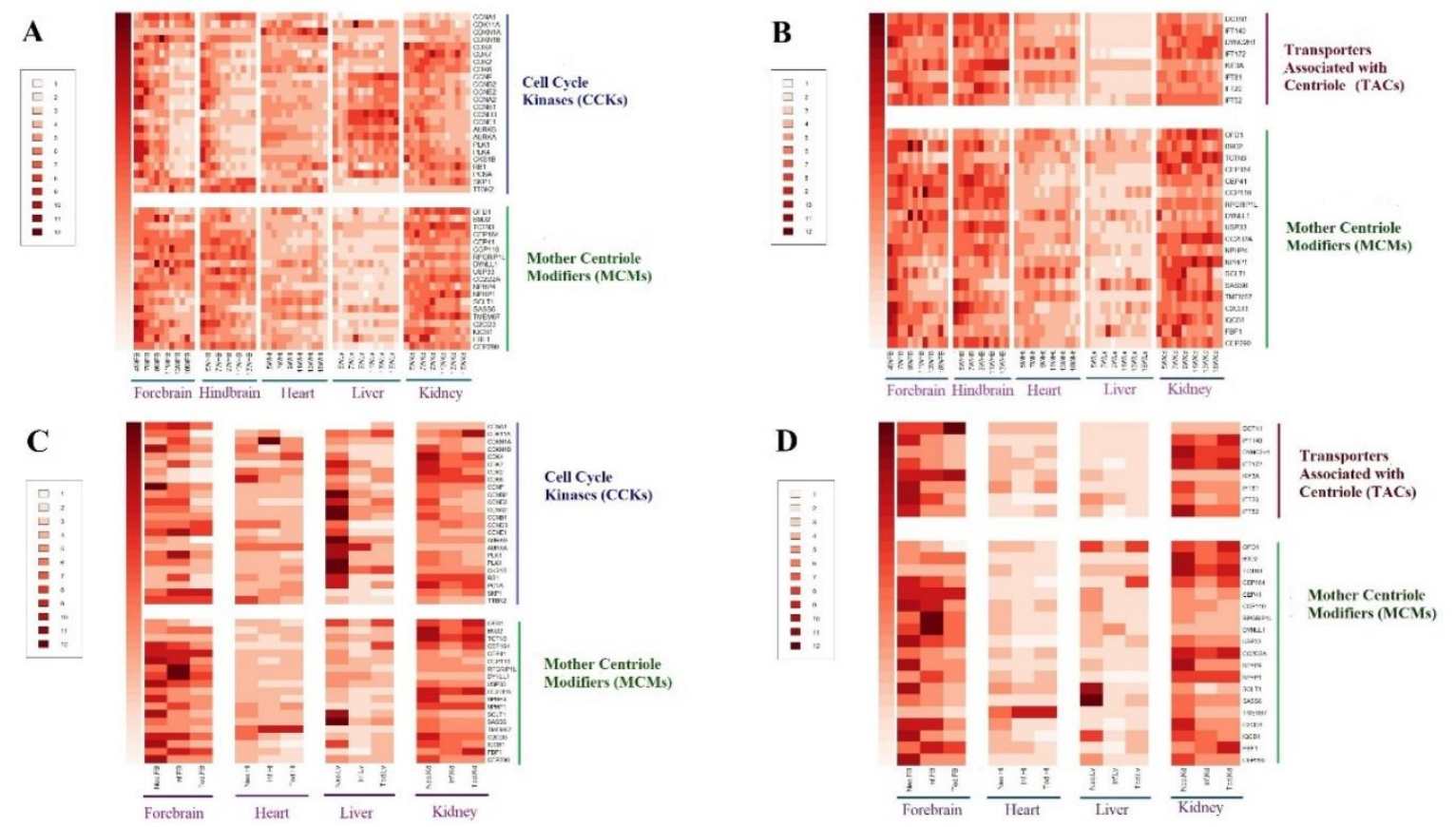

Figure 6. Gene expression levels fluctuate between the cluster of genes involved in cell cycle and ciliogenesis. Baseline expression of cell cycle kinases, mother centriole modifiers and transporters associated with centriole specific genes in brain, heart, liverand kidney in different developmental stages of humans. Heat map A and heat map B are depicting the developmental stages of embryo in weeks and heat map $\mathrm{C}$ and heat map D are representing the developmental stages for neonate, infant, toddler with brain, heart, liver and kidney specific expression (from left to right clusters). The baseline expression level of these genes with their FPKM (Fragments Per Kilobase of transcript per Million mapped reads) values has been collected from "Human RNA-seq time-series of the development of seven major organs"; Expression Atlas (http://www.ebi.ac.uk/gxa; Ref: Expression Atlas update - an integrated database of gene and protein expression in humans, animals and plants; Nucleic Acids Research, 2016). Expression level is proportional to red color intensity in Heat Map. The Heat Map has been drawn in R Studio (version 3.6.2).

How a ciliated cell resumes cell cycle? It requires activation of signaling to reabsorb the cilium for retrieving centriole. What should be the signal to resume cell cycle? It could be again expression of a milestone determining TF. Once such a TF has been modulated during development, the cell can dismantle cilia. It is quite possible that temporal expression of TFs determines this event because the TFs are differentially expressed in various tissues. How TF expression is temporally and spatially regulated remains a dogmatic concept. It cannot be explained solely by the genetic and epigenetic mechanisms. Nevertheless, we can intuit the idea that a TF with binary decision making can switch the role of centriole between cell cycle and ciliogenesis with the help of a protein-interaction network. Once we can identify any master regulator of TF expression, it may reveal yet another level of control for producing the cell mass and their efficient use in the organ development and body structure. There may exist a binary function control integrated with a feedback loop. The feedback part may be able to repeat routine signaling. However, how the linear non-repeating and repeating cellular functions are integrated is not well understood. Further, the fine tuning of cellular layout for body plan may require additional level of molecular sensors to determine what is the limit of growth in normal cells, which are part of an organ.

\subsection{What it Should Accomplish and Abstain?}

The putative molecule should sense cell density, integrate cellular signaling and intercept mechano-transduction to initiate organization of the cell mass. It should also perceive completion of a phase of cellular organization in the niche to signal a halt. Furthermore, such a molecule should be able to coordinate triggering of a new $\mathrm{TF}$ to switch over function or resume proliferation for the 
progressing of organization to a new phase. It should not be simultaneously modulating and driving two conflicting actions like cell division and cell organization. In other words, the candidate molecule should work as a switchable TF and perform a binary action. Another attribute it should be having is that a threshold sensing system rather than all or none. The predicted molecular interactome (Figure 3) harbors signaling proteins, TFs, mechano-transduction sensors, developmental regulators and protein modifiers. The dynamism of protein-protein interaction network is imperative of controlling cell cycle and centriole synthesis.

\subsection{What is the Modular Nature of These Proteins?}

Many key proteins involved in the early development of the centriole have the C2 domain. This domain was first identified in a protein kinase C [129]. Protein kinases are a major family of proteins that regulate the cell cycle. The $\mathrm{C} 2$ domain proteins also function in signal transduction and membrane trafficking, which are essential processes for cilia biogenesis and maintenance. C2 domain proteins are known to bind calcium and phospholipids [130]. Calcium plays a key role during cellular and ciliary signaling. Since the $\mathrm{C} 2$ domain proteins are common in the cell cycle and ciliogenesis, as the key proteins utilized for centriole biogenesis and ciliogenesis, we hypothesize that some $\mathrm{C} 2$ domain containing proteins are key molecules linking the signal switching between the cell division and ciliogenesis. Many research findings substantiate the role of $\mathrm{C} 2$ domain proteins in cilia. For example, B9 domain proteins are key components of Meckel Gruber Syndrome (MKS) complex and possess a distinct C2 domain. The proteins with B9 domain e.g. MKS1, B9D1 and B9D2 are exclusive in the ciliated organisms [131] and are localized to the transition zone of the basal body $[132,133]$. B9D1 is essential for localization of proteins like Arl13b and Adenyl cyclase III (ACIII) required in ciliogenesis. In addition, B9D1 is required for proper Hh signal transduction. In B9D1 mutant mice the ciliary protein composition was abnormal, likely due to intra-cellular signaling abnormalities. Similarly, MKS1 was also identified in the basal body proteome suggesting a role in cilia. However, its presence in the centrosomes in non-ciliated cells indicates a non-cilia function too, perhaps like in centriolar migration [134]. This protein could shuttle between a process before cell division and ciliogenesis, respectively. Many proteins with C2 domain are involved in membrane trafficking, a step critical for organelle development and growth of cells like in neurites.

The Decider Of CytoKinesis (DOCK) family of proteins have C2 domain and are involved in the regulation of the small GTPases of Rho family (Rac, Rho and CDC42). Dock/Zizimin are vital for cell division, cell migration, cell adhesion and, apoptosis [135]. Furthermore, there are some C2 domain proteins that are essential for cell cycle regulation. Likewise, PTEN and TENSIN belong to the C2 Tensin-type domain containing proteins. PTEN blocks cell cycle at G1 phase. It grips on PI3K/Akt signaling pathway and halts progression to G1 thereby suppresses tumor. PI3K/Akt pathway is targeted by cyclin-dependent kinase inhibitor p27(KIP1). PTEN inactivation triggers constitutive Akt/PKB signaling pathway activation. It leads to uncontrolled proliferation of cell [136]. Similarly, overexpression of the mammalian tensin activates both the JNK and p38 MAPK pathways that leads to cancer [137]. In summary, the C2 domain containing proteins are the first line of molecules involved in coordinating the signaling event of cell cycle, centriole biogenesis and ciliogenesis. Diverse modules like kinase, phosphatase, proteases are very important for orchestration of cellular fate and development. However, the possibility of other modular proteins' involvement in this regard can not be excluded.

\subsection{The Alternating Pattern of Gene Expression for Production of Cell Mass and Cilia}

The cellular processes are reflected in the relative concentration of proteins present in a cell. A protein highly required in a process is transcribed more than a less required protein. Therefore, the RNAs of respective proteins shows differential expression in the cells. It can be detected by profiling the global RNA (transcriptome) in a cell or tissue by microarray and RNA sequencing (RNAseq). The latter is a technique advanced by Next Generation Sequencing (NGS) The analysis of the cellular transcriptome reveals temporal and locus specific changes in the expression of genes when a cell responds to environmental stimuli. As a method, RNAseq can be applied to gather insights about 
several scientific questions related to gene expression profiling, disease associated alternate splicing events and single nucleotide polymorphisms manifesting in disease [138]. RNAseq also reveals transcriptional regulation of cellular signalling pathways, centriole biogenesis, cell cycle and ciliogenesis during metazoan development. The expression levels of the genes related to transporters associated with centrioles, mother centriole modifiers and cell cycle kinases etc. are analysed in a tissue specific manner to understand the role of these genes in different developmental stages of the human brain, heart, liver and kidney. The Expression Atlas is a database that offers information on the variable gene expression across different cells, tissues, organs, developmental stage and disease conditions in animal and plant samples [139]. We have analyzed data retrieved from expression atlas (Human RNA-seq time-series of the development of seven major organs, https://www.ebi.ac.uk/gxa/experiments/E-MTAB-6814/Results) to understand the switching on and off of various genes associated with cell cycle (Cell Cycle Kinases, CCKs), with centrioles (Mother Centriole Modifiers, MCM) and Transporters Associated with Centrioles (TACs). The microarray and RNAseq are experimental data that have been manually curated and annotated according to gene ontology (GO) terms. Analysis of the data, represented as heat map (Figure 6) displays the very dynamic changes in the expression level of the genes in a temporal fashion across the tissues. We could not discern whether the dynamic changes in expression of genes are totally offset between the various clusters of genes. Total offset of the gene expression in functional clusters is also not expected. Nevertheless, the asynchronous fluctuations in the gene expression in different organs are an indication that the body plan is evolving in a differential manner in each tissue. To understand how the master plan of body architecture is coordinated more detailed sampling of global gene expression data, and a temporal progression map are required. It will need tools like advanced genomics, transcriptomics, proteomics and big data computing power and sophisticated bioinformatics tools. The future will certainly reveal more secrets of the genetic architecture of complex body plan design and execution in metazoan.

\section{Conclusions}

The centrioles link two key events in metazoan development: cell division and ciliogenesis. The regulation of cell division is required for proper development and to avoid development of defcts and malignancies. Upon completion of the cell cycle, the centriole is retrieved from cell cycle to initiate ciliogenesis, a step before organogenesis. The organogenesis directed through developmental pathways are hosted in the ciliary compartment in most tissues of the body. These signaling pathways, channelized through cilia, delineate the body plan of the metazoans. Thus, centriole serves as a pivot to alternatively switch between cell division and body plan elaboration. In this grand programing and scene alterations, the centriole utilizes few key proteins with specialized domain modules like $\mathrm{C} 2$, phosphatases, proteases and kinases that can turn on and off the signaling pathways involved in the cell division and ciliogenesis. The existence of a grand regulator of such a switch could be speculated but its molecular identity is still elusive. Regardless of the regulators and its role, the centriole remains as the key organelle that serves as the anvil to mold the molecular design of the grandeur structural complexity displayed in metazoans.

Author Contributions: SV conceived the manuscript, ROJ written the initial draft. SK conceived the gene expressionanalysis. A written the gene expression analysis. SC developed expression data images. All authors edited to develop the final manuscript.

Funding: ROJ is supported by CSIR-JRF fellowship by Government of India. SV is supported by DBT-Ramalingaswami Re-Entry Fellowship (BT/HRD/35/02/2006 dt. 4/11/2015), Department of Biotechnology, Ministry of Science and Technology, Government of India. A, SC are supported by Department of Biotechnology, and Ministry of Science and Technology, Government of India, respectively (SR/NM/NB-1087/2017). SK is supported by DBT-IYBA, Government of India (BT 101 O/IYBAJ20 16/02).

Acknowledgments: The authors acknowledge EMBL for permitting use of EMBL expression atlas data.

\section{References}


1. Martindale, M.Q., The evolution of metazoan axial properties. Nat Rev Genet, 2005. 6(12): p. 917-27.

2. Brooke, N.M., J. Garcia-Fernandez, and P.W. Holland, The ParaHox gene cluster is an evolutionary sister of the Hox gene cluster. Nature, 1998. 392(6679): p. 920-2.

3. Creutz, C.E., et al., The copines, a novel class of $\mathrm{C} 2$ domain-containing, calcium-dependent, phospholipid-binding proteins conserved from Paramecium to humans. J Biol Chem, 1998. 273(3): p. 1393-402.

4. Aires, R., A. Dias, and M. Mallo, Deconstructing the molecular mechanisms shaping the vertebrate body plan. Curr Opin Cell Biol, 2018. 55: p. 81-86.

5. Zatulovskiy, E., et al., Cell growth dilutes the cell cycle inhibitor Rb to trigger cell division. Science, 2020. 369(6502): p. 466-471.

6. Ishikawa, H. and W.F. Marshall, Ciliogenesis: building the cell's antenna. Nat Rev Mol Cell Biol, 2011. 12(4): p. 222-34.

7. Tse, L.H. and Y.H. Wong, GPCRs in Autocrine and Paracrine Regulations. Front Endocrinol (Lausanne), 2019. 10: p. 428.

8. Fliegauf, M., T. Benzing, and H. Omran, When cilia go bad: cilia defects and ciliopathies. Nat Rev Mol Cell Biol, 2007. 8(11): p. 880-93.

9. Lim, S. and P. Kaldis, Cdks, cyclins and CKIs: roles beyond cell cycle regulation. Development, 2013. 140(15): p. 3079-93.

10. Wloga, D., E. Joachimiak, and H. Fabczak, Tubulin Post-Translational Modifications and Microtubule Dynamics. Int J Mol Sci, 2017. 18(10).

11. Khoury, G.A., R.C. Baliban, and C.A. Floudas, Proteome-wide post-translational modification statistics: frequency analysis and curation of the swiss-prot database. Sci Rep, 2011. 1.

12. Humphrey, S.J., D.E. James, and M. Mann, Protein Phosphorylation: A Major Switch Mechanism for Metabolic Regulation. Trends Endocrinol Metab, 2015. 26(12): p. 676-687.

13. Dephoure, N., et al., A quantitative atlas of mitotic phosphorylation. Proc Natl Acad Sci U S A, 2008. 105(31): p. 10762-7.

14. Olsen, J.V., et al., Quantitative phosphoproteomics reveals widespread full phosphorylation site occupancy during mitosis. Sci Signal, 2010. 3(104): p. ra3.

15. Lee, S.Y., C. Jang, and K.A. Lee, Polo-like kinases (plks), a key regulator of cell cycle and new potential target for cancer therapy. Dev Reprod, 2014. 18(1): p. 65-71.

16. Malumbres, M., Cyclin-dependent kinases. Genome Biol, 2014. 15(6): p. 122.

17. Krasinska, L. and D. Fisher, Non-Cell Cycle Functions of the CDK Network in Ciliogenesis: Recycling the Cell Cycle Oscillator. Bioessays, 2018. 40(6): p. e1800016.

18. Hinchcliffe, E.H., et al., Requirement of Cdk2-cyclin E activity for repeated centrosome reproduction in Xenopus egg extracts. Science, 1999. 283(5403): p. 851-4. 
19. Matsumoto, Y., K. Hayashi, and E. Nishida, Cyclin-dependent kinase 2 (Cdk2) is required for centrosome duplication in mammalian cells. Curr Biol, 1999. 9(8): p. 429-32.

20. Meraldi, P., et al., Centrosome duplication in mammalian somatic cells requires E2F and Cdk2-cyclin A. Nat Cell Biol, 1999. 1(2): p. 88-93.

21. Matsumoto, Y. and J.L. Maller, A centrosomal localization signal in cyclin E required for Cdk2-independent S phase entry. Science, 2004. 306(5697): p. 885-8.

22. Chen, Z., et al., CP110, a cell cycle-dependent CDK substrate, regulates centrosome duplication in human cells. Dev Cell, 2002. 3(3): p. 339-50.

23. Okuda, M., et al., Nucleophosmin/B23 is a target of CDK2/cyclin E in centrosome duplication. Cell, 2000. 103(1): p. 127-40.

24. Lavecchia, A., C. Di Giovanni, and E. Novellino, CDC25A and B dual-specificity phosphatase inhibitors: potential agents for cancer therapy. Curr Med Chem, 2009. 16(15): p. 1831-49.

25. Toyoshima-Morimoto, F., E. Taniguchi, and E. Nishida, Plk1 promotes nuclear translocation of human Cdc25C during prophase. EMBO Rep, 2002. 3(4): p. 341-8.

26. Krasinska, L., et al., Protein phosphatase 2A controls the order and dynamics of cell-cycle transitions. Mol Cell, 2011. 44(3): p. 437-50.

27. Sherr, C.J., et al., D-type cyclins and their cyclin-dependent kinases: G1 phase integrators of the mitogenic response. Cold Spring Harb Symp Quant Biol, 1994. 59: p. 11-9.

28. Aprelikova, O., Y. Xiong, and E.T. Liu, Both p16 and p21 families of cyclin-dependent kinase (CDK) inhibitors block the phosphorylation of cyclin-dependent kinases by the CDK-activating kinase. J Biol Chem, 1995. 270(31): p. 18195-7.

29. Toyoshima, H. and T. Hunter, p27, a novel inhibitor of G1 cyclin-Cdk protein kinase activity, is related to p21. Cell, 1994. 78(1): p. 67-74.

30. Furuno, N., N. den Elzen, and J. Pines, Human cyclin A is required for mitosis until mid prophase. J Cell Biol, 1999. 147(2): p. 295-306.

31. Riabowol, K., et al., The cdc2 kinase is a nuclear protein that is essential for mitosis in mammalian cells. Cell, 1989. 57(3): p. 393-401.

32. Castro, A., et al., The anaphase-promoting complex: a key factor in the regulation of cell cycle. Oncogene, 2005. 24(3): p. 314-25.

33. Hwang, H.C. and B.E. Clurman, Cyclin E in normal and neoplastic cell cycles. Oncogene, 2005. 24(17): p. 2776-86.

34. Spruck, C.H., et al., hCDC4 gene mutations in endometrial cancer. Cancer Res, 2002. 62(16): p. 4535-9.

35. Koepp, D.M., et al., Phosphorylation-dependent ubiquitination of cyclin E by the SCFFbw7 ubiquitin ligase. Science, 2001. 294(5540): p. 173-7.

36. Strohmaier, H., et al., Human F-box protein hCdc4 targets cyclin E for proteolysis and is mutated in a breast cancer cell line. Nature, 2001. 413(6853): p. 316-22. 
37. Welcker, M., et al., Multisite phosphorylation by Cdk2 and GSK3 controls cyclin E degradation. Mol Cell, 2003. 12(2): p. 381-92.

38. Nash, P., et al., Multisite phosphorylation of a CDK inhibitor sets a threshold for the onset of DNA replication. Nature, 2001. 414(6863): p. 514-21.

39. Cizmecioglu, O., et al., Plk2 regulates centriole duplication through phosphorylation-mediated degradation of Fbxw7 (human Cdc4). J Cell Sci, 2012. 125(Pt 4): p. 981-92.

40. Bai, C., et al., SKP1 connects cell cycle regulators to the ubiquitin proteolysis machinery through a novel motif, the F-box. Cell, 1996. 86(2): p. 263-74.

41. Fung, T.K., et al., Cyclin F is degraded during G2-M by mechanisms fundamentally different from other cyclins. J Biol Chem, 2002. 277(38): p. 35140-9.

42. D'Angiolella, V., et al., SCF(Cyclin F) controls centrosome homeostasis and mitotic fidelity through CP110 degradation. Nature, 2010. 466(7302): p. 138-42.

43. Li, J., et al., USP33 regulates centrosome biogenesis via deubiquitination of the centriolar protein CP110. Nature, 2013. 495(7440): p. 255-9.

44. Han, K.J., et al., Deubiquitylase USP9X maintains centriolar satellite integrity by stabilizing pericentriolar material 1 protein. J Cell Sci, 2019. 132(2).

45. Schimmel, J., et al., Uncovering SUMOylation dynamics during cell-cycle progression reveals FoxM1 as a key mitotic SUMO target protein. Mol Cell, 2014. 53(6): p. 1053-66.

46. Li, Y., et al., SUMOylation of the small GTPase ARL-13 promotes ciliary targeting of sensory receptors. J Cell Biol, 2012. 199(4): p. 589-98.

47. Martinez-Alonso, D. and M. Malumbres, Mammalian cell cycle cyclins. Semin Cell Dev Biol, 2020 .

48. Archambault, V. and D.M. Glover, Polo-like kinases: conservation and divergence in their functions and regulation. Nat Rev Mol Cell Biol, 2009. 10(4): p. 265-75.

49. de Carcer, G., G. Manning, and M. Malumbres, From Plk1 to Plk5: functional evolution of polo-like kinases. Cell Cycle, 2011. 10(14): p. 2255-62.

50. Lane, H.A. and E.A. Nigg, Antibody microinjection reveals an essential role for human polo-like kinase 1 (Plk1) in the functional maturation of mitotic centrosomes. J Cell Biol, 1996. 135(6 Pt 2): p. 1701-13.

51. Llamazares, S., et al., polo encodes a protein kinase homolog required for mitosis in Drosophila. Genes Dev, 1991. 5(12A): p. 2153-65.

52. Litvak, V., et al., Mitotic phosphorylation of the peripheral Golgi protein Nir2 by Cdk1 provides a docking mechanism for Plk1 and affects cytokinesis completion. Mol Cell, 2004. 14(3): p. 319-30.

53. Neef, R., et al., Phosphorylation of mitotic kinesin-like protein 2 by polo-like kinase 1 is required for cytokinesis. J Cell Biol, 2003. 162(5): p. 863-75.

54. Ma, S., J. Charron, and R.L. Erikson, Role of Plk2 (Snk) in mouse development and cell proliferation. Mol Cell Biol, 2003. 23(19): p. 6936-43. 
55. Cizmecioglu, O., et al., Plk2 regulated centriole duplication is dependent on its localization to the centrioles and a functional polo-box domain. Cell Cycle, 2008. 7(22): p. 3548-55.

56. Warnke, S., et al., Polo-like kinase- 2 is required for centriole duplication in mammalian cells. Curr Biol, 2004. 14(13): p. 1200-7.

57. Bahassi el, M., et al., Mammalian Polo-like kinase 3 (Plk3) is a multifunctional protein involved in stress response pathways. Oncogene, 2002. 21(43): p. 6633-40.

58. Xie, S., et al., Plk3 functionally links DNA damage to cell cycle arrest and apoptosis at least in part via the p53 pathway. J Biol Chem, 2001. 276(46): p. 43305-12.

59. Sillibourne, J.E. and M. Bornens, Polo-like kinase 4: the odd one out of the family. Cell Div, 2010. 5: p. 25.

60. Duensing, A., et al., Centriole overduplication through the concurrent formation of multiple daughter centrioles at single maternal templates. Oncogene, 2007. 26(43): p. 6280-8.

61. Bettencourt-Dias, M., et al., SAK/PLK4 is required for centriole duplication and flagella development. Curr Biol, 2005. 15(24): p. 2199-207.

62. Habedanck, R., et al., The Polo kinase Plk4 functions in centriole duplication. Nat Cell Biol, 2005. 7(11): p. 1140-6.

63. Peel, N., et al., Overexpressing centriole-replication proteins in vivo induces centriole overduplication and de novo formation. Curr Biol, 2007. 17(10): p. 834-43.

64. Macmillan, J.C., et al., Comparative expression of the mitotic regulators SAK and PLK in colorectal cancer. Ann Surg Oncol, 2001. 8(9): p. 729-40.

65. Ko, M.A., et al., Plk4 haploinsufficiency causes mitotic infidelity and carcinogenesis. Nat Genet, 2005. 37(8): p. 883-8.

66. Basto, R., et al., Centrosome amplification can initiate tumorigenesis in flies. Cell, 2008. 133(6): p. 1032-42.

67. Fode, C., C. Binkert, and J.W. Dennis, Constitutive expression of murine Sak-a suppresses cell growth and induces multinucleation. Mol Cell Biol, 1996. 16(9): p. 4665-72.

68. Cunha-Ferreira, I., et al., The SCF/Slimb ubiquitin ligase limits centrosome amplification through degradation of SAK/PLK4. Curr Biol, 2009. 19(1): p. 43-9.

69. Korzeniewski, N., et al., Cullin 1 functions as a centrosomal suppressor of centriole multiplication by regulating polo-like kinase 4 protein levels. Cancer Res, 2009. 69(16): p. 6668-75.

70. Rogers, G.C., et al., The SCF Slimb ubiquitin ligase regulates Plk4/Sak levels to block centriole reduplication. J Cell Biol, 2009. 184(2): p. 225-39.

71. Guardavaccaro, D., et al., Control of meiotic and mitotic progression by the $\mathrm{F}$ box protein beta-Trcp1 in vivo. Dev Cell, 2003. 4(6): p. 799-812.

72. Holland, A.J., et al., Polo-like kinase 4 kinase activity limits centrosome overduplication by autoregulating its own stability. J Cell Biol, 2010. 188(2): p. 191-8. 
73. Guderian, G., et al., Plk4 trans-autophosphorylation regulates centriole number by controlling betaTrCP-mediated degradation. J Cell Sci, 2010. 123(Pt 13): p. 2163-9.

74. Sillibourne, J.E., et al., Autophosphorylation of polo-like kinase 4 and its role in centriole duplication. Mol Biol Cell, 2010. 21(4): p. 547-61.

75. Gautschi, O., et al., Aurora kinases as anticancer drug targets. Clin Cancer Res, 2008. 14(6): p. 1639-48.

76. Kufer, T.A., et al., Human TPX2 is required for targeting Aurora-A kinase to the spindle. J Cell Biol, 2002. 158(4): p. 617-23.

77. Zhu, C., E. Bossy-Wetzel, and W. Jiang, Recruitment of MKLP1 to the spindle midzone/midbody by INCENP is essential for midbody formation and completion of cytokinesis in human cells. Biochem J, 2005. 389(Pt 2): p. 373-81.

78. Winkles, J.A. and G.F. Alberts, Differential regulation of polo-like kinase 1, 2, 3, and 4 gene expression in mammalian cells and tissues. Oncogene, 2005. 24(2): p. 260-6.

79. Macurek, L., et al., Polo-like kinase-1 is activated by aurora A to promote checkpoint recovery. Nature, 2008. 455(7209): p. 119-23.

80. Carmena, M., et al., The chromosomal passenger complex activates Polo kinase at centromeres. PLoS Biol, 2012. 10(1): p. e1001250.

81. Qian, Y.W., E. Erikson, and J.L. Maller, Mitotic effects of a constitutively active mutant of the Xenopus polo-like kinase Plx1. Mol Cell Biol, 1999. 19(12): p. 8625-32.

82. Jang, Y.J., et al., Phosphorylation of threonine 210 and the role of serine 137 in the regulation of mammalian polo-like kinase. J Biol Chem, 2002. 277(46): p. 44115-20.

83. Sulimenko, V., et al., Regulation of microtubule nucleation mediated by gamma-tubulin complexes. Protoplasma, 2017. 254(3): p. 1187-1199.

84. Albert, R. and R.S. Wang, Discrete dynamic modeling of cellular signaling networks. Methods Enzymol, 2009. 467: p. 281-306.

85. Basson, M.A., Signaling in cell differentiation and morphogenesis. Cold Spring Harb Perspect Biol, 2012. 4(6).

86. Nigg, E.A. and A.J. Holland, Once and only once: mechanisms of centriole duplication and their deregulation in disease. Nat Rev Mol Cell Biol, 2018. 19(5): p. 297-312.

87. Azimzadeh, J. and W.F. Marshall, Building the centriole. Curr Biol, 2010. 20(18): p. R816-25.

88. Nigg, E.A. and J.W. Raff, Centrioles, centrosomes, and cilia in health and disease. Cell, 2009. 139(4): p. 663-78.

89. Vorobjev, I.A. and S. Chentsov Yu, Centrioles in the cell cycle. I. Epithelial cells. J Cell Biol, 1982. 93(3): p. 938-49.

90. Franck, N., et al., CDK11(p58) is required for centriole duplication and Plk4 recruitment to mitotic centrosomes. PLoS One, 2011. 6(1): p. e14600. 
91. Kim, T.S., et al., Hierarchical recruitment of Plk4 and regulation of centriole biogenesis by two centrosomal scaffolds, Cep192 and Cep152. Proc Natl Acad Sci U S A, 2013. 110(50): p. E4849-57.

92. Tsou, M.F. and T. Stearns, Mechanism limiting centrosome duplication to once per cell cycle. Nature, 2006. 442(7105): p. 947-51.

93. Kitagawa, D., et al., Structural basis of the 9-fold symmetry of centrioles. Cell, 2011. 144(3): p. 364-75.

94. Nakazawa, Y., et al., SAS-6 is a cartwheel protein that establishes the 9-fold symmetry of the centriole. Curr Biol, 2007. 17(24): p. 2169-74.

95. Bornens, M., Centrosome composition and microtubule anchoring mechanisms. Curr Opin Cell Biol, 2002. 14(1): p. 25-34.

96. Bornens, M. and M. Piel, Centrosome inheritance: birthright or the privilege of maturity? Curr Biol, 2002. 12(2): p. R71-3.

97. Hori, A., et al., A non-canonical function of Plk4 in centriolar satellite integrity and ciliogenesis through PCM1 phosphorylation. EMBO Rep, 2016. 17(3): p. 326-37.

98. Kobayashi, T. and B.D. Dynlacht, Regulating the transition from centriole to basal body. J Cell Biol, 2011. 193(3): p. 435-44.

99. Veleri, S., et al., Ciliopathy-associated gene Cc2d2a promotes assembly of subdistal appendages on the mother centriole during cilia biogenesis. Nat Commun, 2014. 5: p. 4207.

100. Goetz, S.C., K.F. Liem, Jr., and K.V. Anderson, The spinocerebellar ataxia-associated gene Tau tubulin kinase 2 controls the initiation of ciliogenesis. Cell, 2012. 151(4): p. 847-858.

101. Kinzel, D., et al., Pitchfork regulates primary cilia disassembly and left-right asymmetry. Dev Cell, 2010. 19(1): p. 66-77.

102. Hubbert, C., et al., HDAC6 is a microtubule-associated deacetylase. Nature, 2002. 417(6887): p. 455-8.

103. Bettencourt-Dias, M. and D.M. Glover, Centrosome biogenesis and function: centrosomics brings new understanding. Nat Rev Mol Cell Biol, 2007. 8(6): p. 451-63.

104. Michaud, E.J. and B.K. Yoder, The primary cilium in cell signaling and cancer. Cancer Res, 2006. 66(13): p. 6463-7.

105. Overgaard, C.E., et al., Deciliation is associated with dramatic remodeling of epithelial cell junctions and surface domains. Mol Biol Cell, 2009. 20(1): p. 102-13.

106. Pugacheva, E.N., et al., HEF1-dependent Aurora A activation induces disassembly of the primary cilium. Cell, 2007. 129(7): p. 1351-63.

107. Bhogaraju, S., et al., Molecular basis of tubulin transport within the cilium by IFT74 and IFT81. Science, 2013. 341(6149): p. 1009-12.

108. Li, A., et al., Ciliary transition zone activation of phosphorylated Tctex-1 controls ciliary resorption, S-phase entry and fate of neural progenitors. Nat Cell Biol, 2011. 13(4): p. 402-11. 
109. Pugacheva, E.N. and E.A. Golemis, HEF1-aurora A interactions: points of dialog between the cell cycle and cell attachment signaling networks. Cell Cycle, 2006. 5(4): p. 384-91.

110. Shearer, R.F. and D.N. Saunders, Regulation of primary cilia formation by the ubiquitin-proteasome system. Biochem Soc Trans, 2016. 44(5): p. 1265-1271.

111. Passmore, L.A. and D. Barford, Getting into position: the catalytic mechanisms of protein ubiquitylation. Biochem J, 2004. 379(Pt 3): p. 513-25.

112. Schnell, J.D. and L. Hicke, Non-traditional functions of ubiquitin and ubiquitin-binding proteins. J Biol Chem, 2003. 278(38): p. 35857-60.

113. Gerhardt, C., et al., The transition zone protein Rpgrip1l regulates proteasomal activity at the primary cilium. J Cell Biol, 2015. 210(1): p. 115-33.

114. Kim, J., et al., Functional genomic screen for modulators of ciliogenesis and cilium length. Nature, 2010. 464(7291): p. 1048-51.

115. Hilgendorf, K.I., C.T. Johnson, and P.K. Jackson, The primary cilium as a cellular receiver: organizing ciliary GPCR signaling. Curr Opin Cell Biol, 2016. 39: p. 84-92.

116. Bachmann, V.A., et al., Gpr161 anchoring of PKA consolidates GPCR and cAMP signaling. Proc Natl Acad Sci U S A, 2016. 113(28): p. 7786-91.

117. Porpora, M., et al., Counterregulation of cAMP-directed kinase activities controls ciliogenesis. Nat Commun, 2018. 9(1): p. 1224.

118. Singla, V. and J.F. Reiter, The primary cilium as the cell's antenna: signaling at a sensory organelle. Science, 2006. 313(5787): p. 629-33.

119. Satir, P. and S.T. Christensen, Structure and function of mammalian cilia. Histochem Cell Biol, 2008. 129(6): p. 687-93.

120. Wheway, G., D.A. Parry, and C.A. Johnson, The role of primary cilia in the development and disease of the retina. Organogenesis, 2014. 10(1): p. 69-85.

121. Hartill, V., et al., Meckel-Gruber Syndrome: An Update on Diagnosis, Clinical Management, and Research Advances. Front Pediatr, 2017. 5: p. 244.

122. Waters, A.M. and P.L. Beales, Ciliopathies: an expanding disease spectrum. Pediatr Nephrol, 2011. 26(7): p. 1039-56.

123. Zeller, R., J. Lopez-Rios, and A. Zuniga, Vertebrate limb bud development: moving towards integrative analysis of organogenesis. Nat Rev Genet, 2009. 10(12): p. 845-58.

124. Yang, Y. and L. Niswander, Interaction between the signaling molecules WNT7a and SHH during vertebrate limb development: dorsal signals regulate anteroposterior patterning. Cell, 1995. 80(6): p. 939-47.

125. Litingtung, Y., et al., Shh and Gli3 are dispensable for limb skeleton formation but regulate digit number and identity. Nature, 2002. 418(6901): p. 979-83.

126. Finkelstein, R. and N. Perrimon, The orthodenticle gene is regulated by bicoid and torso and specifies Drosophila head development. Nature, 1990. 346(6283): p. 485-8. 
127. Simeone, A., Otx 1 and Otx2 in the development and evolution of the mammalian brain. EMBO J, 1998. 17(23): p. 6790-8.

128. Mears, A.J., et al., Nrl is required for rod photoreceptor development. Nat Genet, 2001. 29(4): p. $447-52$.

129. Takai, Y., et al., Calcium-dependent activation of a multifunctional protein kinase by membrane phospholipids. J Biol Chem, 1979. 254(10): p. 3692-5.

130. Rizo, J. and T.C. Sudhof, C2-domains, structure and function of a universal Ca2+-binding domain. J Biol Chem, 1998. 273(26): p. 15879-82.

131. Bialas, N.J., et al., Functional interactions between the ciliopathy-associated Meckel syndrome 1 (MKS1) protein and two novel MKS1-related (MKSR) proteins. J Cell Sci, 2009. 122(Pt 5): p. 611-24.

132. Dowdle, W.E., et al., Disruption of a ciliary B9 protein complex causes Meckel syndrome. The American Journal of Human Genetics, 2011. 89(1): p. 94-110.

133. Dowdle, W.E., et al., Disruption of a ciliary B9 protein complex causes Meckel syndrome. Am J Hum Genet, 2011. 89(1): p. 94-110.

134. Dawe, H.R., et al., The Meckel-Gruber Syndrome proteins MKS1 and meckelin interact and are required for primary cilium formation. Hum Mol Genet, 2007. 16(2): p. 173-86.

135. Pakes, N.K., D.M. Veltman, and R.S. Williams, Zizimin and Dock guanine nucleotide exchange factors in cell function and disease. Small GTPases, 2013. 4(1): p. 22-7.

136. Steelman, L.S., et al., Contributions of the Raf/MEK/ERK, PI3K/PTEN/Akt/mTOR and Jak/STAT pathways to leukemia. Leukemia, 2008. 22(4): p. 686-707.

137. Katz, B.Z., et al., Tensin can induce JNK and p38 activation. Biochem Biophys Res Commun, 2000. 272(3): p. 717-20.

138. Han, Y., et al., Advanced Applications of RNA Sequencing and Challenges. Bioinform Biol Insights, 2015. 9(Suppl 1): p. 29-46.

139. Petryszak, R., et al., Expression Atlas update--an integrated database of gene and protein expression in humans, animals and plants. Nucleic Acids Res, 2016. 44(D1): p. D746-52. 